\title{
Scientific Hypothesis for Treatment of COVID-19's Lung Lesions by Adjusting ACE/ACE2 Imbalance
}

\author{
F. Ferrara ${ }^{1}$ - A. Vitiello ${ }^{1}(1)$
}

Received: 26 January 2021 / Accepted: 30 March 2021 / Published online: 9 April 2021

(c) The Author(s), under exclusive licence to Springer Science+Business Media, LLC, part of Springer Nature 2021

\begin{abstract}
In March 2019 began the global pandemic COVID-19 caused by the new Coronavirus SARS-CoV-2. The first cases of SARS-CoV-2 infection occurred in November-19 in Wuhan, China. The preventive measures taken did not prevent the rapid spread of the virus to all countries around the world. To date, there are about 2.54 million deaths, effective vaccines are in clinical trials. SARS-CoV-2 uses the ACE-2 protein as an intracellular gateway. ACE-2 is a key component of the Renin Angiotensin (RAS) system, a key regulator of cardiovascular function. Considering the key role of ACE-2 in COVID-19 infection, both as an entry receptor and as a protective role, especially for the respiratory tract, and considering the variations of ACE- 2 and ACE during the stages of viral infection, it is clear the important role that the pharmacological regulation of RAS and ACE-2 can assume. This biological knowledge suggests different pharmacological approaches to treat COVID-19 by modulating RAS, ACE-2 and the ACE/ACE2 balance that we describe in this article.
\end{abstract}

Keywords RAS $\cdot$ ACE- $2 \cdot$ COVID-19 $\cdot$ SARS-CoV-2

\section{Introduction}

The new coronavirus, SARS-CoV-2 is responsible for coronavirus disease (COVID-19), the cause of the current global pandemic. To date COVID-19 has caused 2,54 Mln deaths and 114 Mln million people infected. [1] The COVID-19 pandemic represents a health, social and economic challenge for all countries. Some vaccines are showing encouraging results of about $95 \%$ efficacy in late clinical trials. At present, drug treatments for the viral infection are mostly experimental, some antivirals such as remdesivir have shown good efficacy in reducing mortality and healing time. [2, 3] COVID-19 infection is in most cases asymptomatic or slightly symptomatic, however in a small percentage of cases, especially in older people and those with pre-existing conditions, the infection can be severe and in some cases

Handling editor: Dakshesh Patel.

F. Ferrara

francesco.ferrara@uslumbria1.it

A. Vitiello

antonio.vitiello2@uslumbria1.it

1 Pharmaceutical Department, Usl Umbria 1, A.Migliorati street, 06132 Perugia, Italy fatal. $[4,5]$ Although respiratory symptoms are predominant, [6] in the more severe stages of infection, multi-organ dysfunction may occur due to an abnormal and generalized inflammatory response that causes injury to vital organs such as lungs, heart, liver. [7, 8] People with diseases such as diabetes, hypertension, heart disease, are at increased risk of serious complications from COVID-19, suggesting that therapeutic treatment of underlying diseases should not be interrupted during COVID-19 infection. [9, 10] SARSCoV-2 has a high structural homology with SARS-CoV, and also share the same cell entry receptor, the angiotensin conversion enzyme 2 (ACE-2) [11]. ACE-2 is a protein with a key role in the angiotensin renin (RAS) system. RAS is a key regulator of the cardiovascular system. In the more severe stages of COVID-19 infection there is a dysregulation of the RAS system and the ACE/ACE-2 balance, suggesting an important role of RAS and ACE-2 throughout the entire COVID-19 infection phase. Important role of RAS and ACE-2 during the entire phase of COVID-19 infection. A few months ago the first vaccines against COVID-19 have been authorized, starting a massive vaccination campaign, however the research continues to find therapeutic solutions aimed at avoiding the most serious complications caused by COVID-19 [12-15]. 


\section{Renin Angiotensin System (RAS)}

The renin-angiotensin system (RAS) is a physiological mechanism with a key regulatory role in various functions of the cardiovascular system. RAS is an enzymatic cascade consisting of a "classical way" and a "non-classical way". (Fig. 1) In the main (classical) enzymatic pathway, renin converts angiotensinogen to angiotensin I (Ang I). Ang I is then converted to angiotensin II (Ang II) by the angiotensin conversion enzyme (ACE). In the non-classical enzyme pathway ACE- 2 converts Ang II to angiotensin 1-7 (Ang 1-7) and Ang I to angiotensin 1-9 (Ang 1-9). ACE-2 is expressed in renal, cardiovascular, pulmonary and gastrointestinal tissues. The biological effects of AngII on AT-1r receptors are vasoconstriction and stimulant of aldosterone release; myocardial hypertrophy, interstitial fibrosis, endothelial dysfunction, increased inflammatory state, increased oxidative stress and increased coagulation. Ang II can also cause increased inflammation through the production of IL-6, TNF (Tumor Necrosis Factor) and other inflammatory cytokines. [16, 17] Ang 1-7 and Ang 1-9 have opposite biological effects to Ang II through stimulation of the Mas receptor (MASr) and Angiotensin type II receptors (AT-2r). MASr are expressed on the surface of the bronchial muscle and alveolar epithelium of the lungs. [18, 19] Ang 1-7 and Ang1-9 have anti-inflammatory, antifibrosis, vasodilation effects. Evidence shows that the RAS system varies in conditions of pathologies such as BPCO or viral infections, suggesting a possible involvement in homeostasis and function of the respiratory system and other ongoing systems of certain diseases. Some data demonstrate the variation of RAS and ACE-2 during COVID-19 infection, in particular an increase of ACE-2 in the early stages of infection and a rapid decrease in the more severe stages. It may be possible that ACE-2 has a protective effect and when it decreases there is a worsening of the inflammatory pulmonary state. [20, 21]

\section{SARS-CoV-2 and RAS}

The expression of ACE2 varies in a time-dependent manner after SARS-CoV 2 infection. The role of ACE-2 in the pathophysiology of SARS-Cov-2 infection is not yet fully understood, because ACE- 2 is not only the virus entry receptor, the modulation and variation of RAS and ACE-2 during the various stages of infection have been demonstrated, and the role of ACE-2 in infected lung tissue is unclear, especially during the more severe stages of infection, where

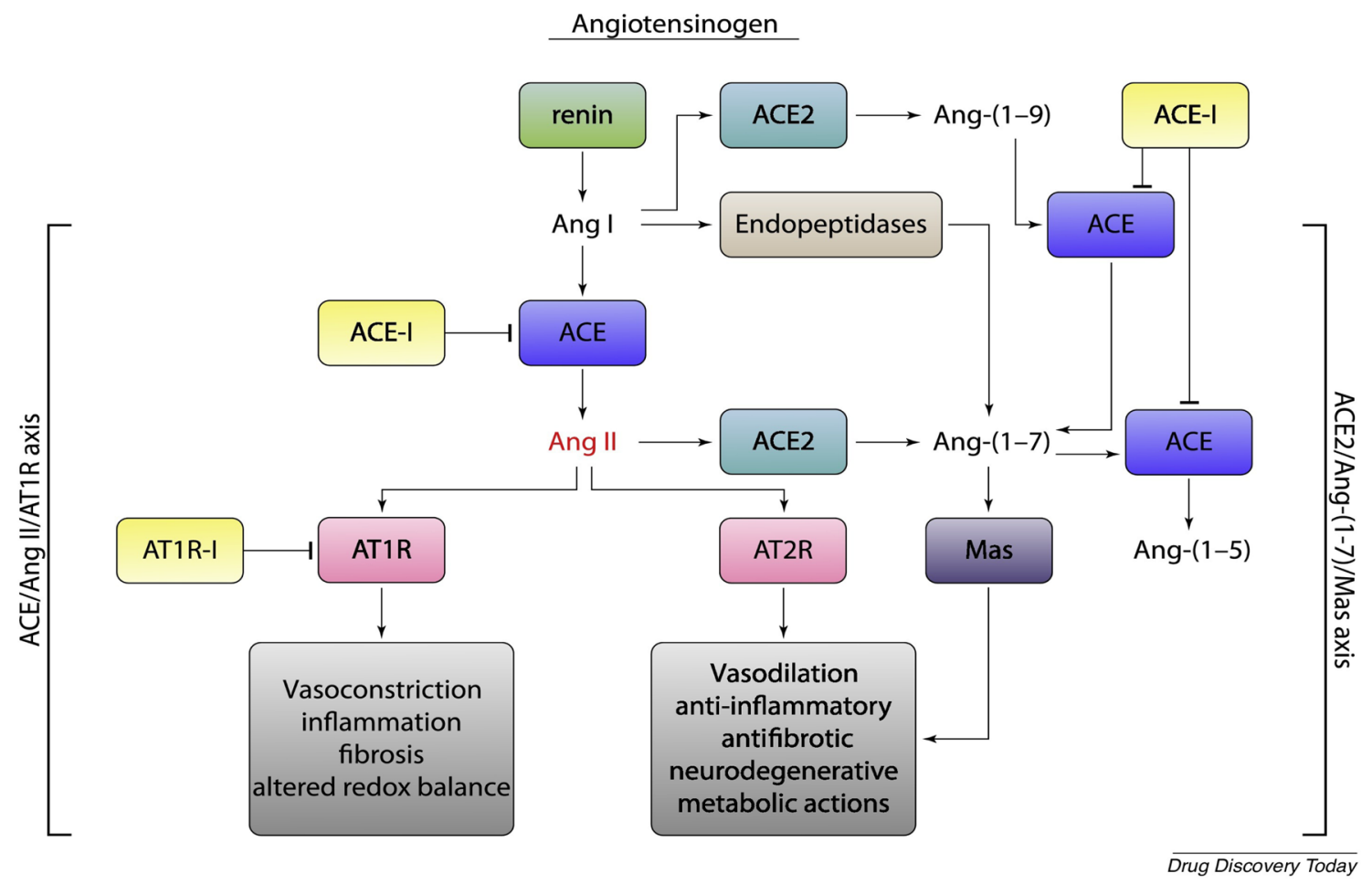

Fig. 1 Schematic representation of the renin-angiotensin system (RAS). Activation of AT2-r and MasR receptors induce vasodilatory, anti-inflammatory, and antifibrotic effects with potential clinical ben- efit in the most severe stages of SARS-CoV-2 infection, in contrast, vasoconstrictor, proinflammatory, and profibrotic effects mediated by AT1-r activation could increase lung and tissue damage 
it appears to have a protective role. Evidence shows that SARS-CoV-2 infection early stages produces decreased expression and activity of ACE-2 in lungs tissue [22]. In addition, during COVID-19 infection there is an increase in circulating Ang II concentrations [23]. This evidence suggests the important role played by RAS during COVID-19 viral infection. In particular, the ACE/ACE-2 imbalance creates a greater activation of the ACE/Ang II/AT1-r axis with increased AT1r stimulation by Ang II with known harmful biological effects, increased inflammation, vasoconstriction, profibrotic activity. The imbalance of RAS and ACE/ ACE-2 ratio suggests potential pharmacological approaches that rebalance the system.

\section{ACE-2}

SARS-CoV-2 affects different tissues of the host organism, this can be explained by the expression of ACE2. In fact, the presence of ACE2 has been detected in the respiratory tract, [24-26] in the myocardium, in the arteries, in the esophagus, in the brain,, in the urinary tract. [27-30]. Depending on the tissue expression of ACE2, it has been hypothesized that organs with higher expression of ACE2 might be at higher risk of damage by COVID-19, also supporting this thesis, it has been found that young people have a lower concentration of ACE2 in the respiratory tract, explaining, at least in part, why they are less likely to have lung injury caused by COVID-19 than older people. Evidence shows that ACE2 expression may vary in dependence of preexisting disease. In idiopathic pulmonary fibrosis, in COPD, ACE2 is upregulated in the lungs probably as a counterregulatory response [31-33] This may partly explain the increased susceptibility of severe COVID-19 infection in patients with pre-existing respiratory tract comorbidity. In addition, ACE2 expression may also partially explain the cardiac, hepatic, and neurological injuries caused [34-37] by COVID-19 induced by endocellular penetration of SARS-CoV-2 and the hyperactive inflammatory state induced in the more severe stages of infection.

\section{ACE/ACE-2 Balance and COVID-19 Infection}

Several evidence shows beneficial effects of ACE-I and ARB (Angiotensin II receptor antagonists) in lung lesions including acute respiratory distress syndrome ARDS and pulmonary fibrosis. [38, 39] In view of these aspects, and the mechanism of action of ACE-i and ARB in decreasing Ang II activity, it may be thought that Ang II plays an important role in lung damage. Some evidence shows that the use of ARB reduces pro-inflammatory cytokine secretion and lung injury [40]. Data from retrospective human studies show that ACE-I can prevent or reduce the severity of pneumonia [41]. In addition, treatment with AT-1(ARB) receptor antagonists in COPD reduces inflammation, comorbidities and complications of the disease [42]. In addition, in vitro studies show that Ang II induces apoptosis in a human epithelial cell line and in rat type II pneumocytes, effects that are blocked by treatment with ACE-I or AT receptor antagonists [43, 44], other studies demonstrate the correlation between over-stimulated AT1r and the promotion of apoptosis in lung epithelial cells [45]. Therefore this evidence shows that Ang II and At-1r stimulation may play a key role in lung lesions. On the other hand, a large amount of evidence associates the stimulation of MASr by Ang (1-7) and Ang (1-9) protective effects in mitigating lung lesions by acting in opposition to the effects of Ang II. Some in vivo evidence indicates that ACE2/Ang axis (1-7) can mitigate pulmonary fibrosis, in fact the treatment with recombinant ACE- 2 reduces the release of pro-fibrotic cytokines and the infiltration of inflammatory cells, reducing lung damage [46]. Some studies associate the anti-inflammatory and antifibrotic effects of Ang (1-7) with the inhibition of signaling cascades involving MAP Kinase and NF- $\mathrm{KB}$ [47]. In view of these biological mechanisms, it is clear that the objective of pharmacological strategies is to restore the ACE1/ACE2 balance in favor of the ACE2/Ang axis(1-7) (Fig. 2).

\section{Pharmacological Strategies}

ACE-i are drugs commonly used for the treatment of various cardiovascular diseases, such as essential hypertension, ischemic heart disease. In view of the above, ACE/Ang II axis inhibition would probably be of therapeutic benefit in reducing COVID-19 lung damage, although to date there is no evidence in the literature. However, ACE inhibition leads to an increase in bradykinin, which can cause coughing and inflammation of the airways, and this is a disadvantage. The use of ARB antagonizes the effects of Ang

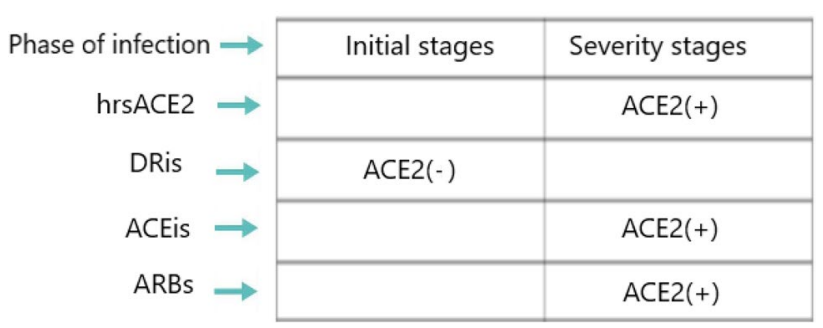

Fig. 2 SARS-CoV-2 uses ACE-2 as a cellular input receptor, decreasing its expression and activity (Zhang et al. 2020), this may favor the ACE/Ang II/AT-1r axis. Therapeutic strategies can rebalance the ACE/ACE-2 balance in favor of the ACE-2/Ang(1-7)MASr axis with antifibrotic and antifibrotic effects in reducing COVID-19 lung lesions 
Table 1 Several clinical trials are underway to test the efficacy of RAS modifying agents in COVID-19 positive patients

\begin{tabular}{ll}
\hline Study title & Status \\
\hline Recombinant bacterial ACE2 receptors -like enzyme of B38-CAP could be promising & Not yet recruiting \\
COVID-19 infection- and lung injury preventing drug better than recombinant human & \\
ACE2 & \\
$\begin{array}{l}\text { Combination of recombinant bacterial ACE2 receptors like enzyme of B38-CAP and } \\
\text { isotretinoin coul be promising Covid 19 infection and lung injury preventing drug }\end{array}$ & Not yet recruiting \\
better than recombinant human ACE & \\
$\begin{array}{l}\text { Impact of Angiotensin II Receptor Blockers treatment in patients with COVID-19 } \\
\text { Switch of Renin-Angiotensin system inhibitors in patients with Covid-19 }\end{array}$ & Recruiting \\
$\begin{array}{l}\text { Renin angiootensin aldosterone system inhibitors,hypertension and Covid-19 } \\
\text { Telmisartan for treatment of Covid-19 patients }\end{array}$ & $\begin{array}{l}\text { Recruiting } \\
\text { Treatment of angiotensin peptide (1-7) for Covid-19 }\end{array}$ \\
$\begin{array}{l}\text { Telmisartan in respiratory failure due to COVID-19 } \\
\text { ACE Inhibitors, Angiotensin II Type-I receptor blockers and severity of COVID-19 }\end{array}$ & Recruiting \\
\hline
\end{tabular}

II on AT-1 receptors. Some evidence shows a decrease in mortality and morbidity in COVID-19 patients treated with ARB prior to admission. [48] Blocking of AT-1 receptors not only decreases the biological effects mediated by AT-1 but may induce a counter-regulation response in the RAS system by increasing the ACE-2/Ang pathway (1-7). An advantage over ACE-i is that the use of ARB does not cause an increase in bradykinin and risk of dry cough. Another therapeutic strategy is the use of direct renin inhibitor (DRI), with an inhibition downstream of the RAS cascade. However, this pharmacological strategy would also block all the beneficial effects of the ACE-2/Ang axis (1-7). An interesting pharmacological approach is the use of soluble ACE2, used as "bait" to bind the SARS-CoV-2 virus and save the cellular ACE2. A recombinant human ACE-2 has been tested and well tolerated in Phase 1 and a Phase 2 study with ARDS patients [49] demonstrating a reduction in circulating levels of Ang II and IL-6. Since the beginning of the COVID-19 pandemic there has been a heated debate in the scientific world whether the use of ACE-i or ARB can increase the risk of COVID-19 infection. To date the evidence does not demonstrate this, and it is recommended not to suspend the current therapeutic treatment. [50, 51] Among the various agents under investigation is soluble recombinant human ACE2 (hrsACE2) with a dual mechanism of action anti COVID-19, 2 binds the viral spike protein neutralizing SARS-CoV-2, 3 and minimize damage to multiple organs, including the lungs, kidneys, and heart, by reducing ang II concentrations and increasing conversion to ang (1-7). In addition, in vitro evidence demonstrates that hrsACE2 can reduce the burden of SARS-CoV-2 by a factor of 1000-5000 by acting as a decoy to effectively neutralize SARS-CoV-2. [52-56] Another novel approach in this direction is the use of ACE2-like enzyme. An example of such agents is B38-CAP. B38-CAP shares structural similarity with $\mathrm{ACE} 2$. In vitro evidence shows that the recombinant B38-CAP protein catalyzes the conversion of angiotensin
II to angiotensin 1-7, as do other known ACE2-targeting peptides. [57] Finally, another approach in this direction is the administration of ang (1-7) derived plasma in COVID19 positive patients. Several clinical studies are ongoing to confirm these hypotheses. (Table 1).

\section{Conclusion}

Evidence shows a strong correlation between RAS and COVID-19 infection, in particular Ang II stimulating the AT-1r receptor could amplify lung damage. Several therapeutic strategies exist to direct ACE/ACE-2 balance toward the ACE-2/Ang axis (1-7) with antifibrotic and vasodilatory effects mediated by stimulation of MASr and reduction of inflammatory and profibrotic effects of Ang II. Clinical studies are needed to generate epidemiological evidence in this direction.

Acknowledgement The authors have nothing to say about ethical standards, ethical approval and funding. This manuscript is not a clinical trial and does not violate ethical rules. No funding has been received for its preparation.

Author Contribution AV Conceptualization, Writing-original draft, Methodology, Writing — original draft. FF Writing_review \& editing, Supervision, Validation.

Data Availability There are no sensitive data and no patients were recruited for this study.

\section{Delarations}

Conflict of interest All Authors declare that they have no conflict of interest to disclose.

Ethical approval The authors certify that the manuscript is original, never submitted to other journal for publication before. All authors contributed equally to the manuscript and had the opportunity to revise and approve the final text. 


\section{References}

1. World health organization (WHO) https://www.who.int/emerg encies/diseases/novel-coronavirus2019/situation-reports (Situation Reports Feb 2021).

2. Ferrara, F., Porta, R., D’Aiuto, V., \& Vitiello, A. (2020). Remdesivir and COVID-19. Irish Journal of Medical Science, 17, 1-2. https://doi.org/10.1007/s11845-020-02401-5.

3. Vitiello, A., \& Ferrara, F. (2020). Remdesivir versus ritonavir/ lopinavir in COVID-19 patients. Irish Journal of Medical Science, 18, 1-2. https://doi.org/10.1007/s11845-020-02440-y.

4. Ruan, Q., Yang, K., Wang, W., Jiang, L., \& Song, J. (2020). Clinical predictors of mortality due to COVID-19 based on an analysis of data of 150 patients from Wuhan. China. Intensive Care Med., 46(5), 846-848. https://doi.org/10.1007/s00134-020-05991-x.

5. Yang, J., Zheng, Y., Gou, X., Pu, K., Chen, Z., Guo, Q., Ji, R., Wang, H., Wang, Y., \& Zhou, Y. (2020). Prevalence of comorbidities and its effects in patients infected with SARS-CoV-2: a systematic review and meta-analysis. International Journal of Infectious Diseases, 94, 91-95. https://doi.org/10.1016/j.ijid.2020. 03.017.

6. Ferrara, F., Granata, G., Pelliccia, C., La Porta, R., \& Vitiello, A. (2020). The added value of pirfenidone to fight inflammation and fibrotic state induced by SARS-CoV-2: Anti-inflammatory and anti-fibrotic therapy could solve the lung complications of the infection? European Journal of Clinical Pharmacology, 76(11), 1615-1618. https://doi.org/10.1007/s00228-020-02947-4.

7. Shi, S., Qin, M., Shen, B., Cai, Y., Liu, T., Yang, F., et al. (2020). Association of cardiac injury with mortality in hospitalized patients with covid-19 in Wuhan China. JAMA Cardiol., 5(7), 802-810. https://doi.org/10.1001/jamacardio.2020.0950.

8. Vitiello, A., Ferrara, F., Pelliccia, C., Granata, G., \& La Porta, R. (2020). Cytokine storm and colchicine potential role in fighting SARS-CoV-2 pneumonia. Italian Journal of Medicine., 14(2), 88-94. https://doi.org/10.4081/itjm.2020.1284.

9. Ferrara, F. (2020). Antirheumatic in SARS-CoV-2: benefit or risk? Italian Journal of Medicine., 14(2), 114-115. https://doi.org/10. 4081/itjm.2020.1290.

10. Guo, T., Fan, Y., Chen, M., Wu, X., Zhang, L., He, T., et al. (2020). Cardiovascular implications of fatal outcomes of patients with coronavirus disease 2019 (COVID-19). JAMA Cardiol., 5(7), 811-818. https://doi.org/10.1001/jamacardio.2020.1017.

11. Walls, A. C., Park, Y. J., Tortorici, M. A., Wall, A., McGuire, A. T., \& Veesler, D. (2020). Structure, function, and antigenicity of the SARS-CoV-2 spike glycoprotein. Cell, 181, 894-904.

12. Vitiello, V., \& Ferrara, F. (2021). Colchicine and SARS-CoV-2 Management of the hyperinflammatory state. Respiratory Medicine, 178, 106322. https://doi.org/10.1016/j.rmed.2021.106322.

13. Vitiello, A., La Porta, R., D'Aiuto, V., \& Ferrara, F. (2021). Pharmacological approach for the reduction of inflammatory and prothrombotic hyperactive state in COVID-19 positive patients by acting on complement cascade. Human Immunology. https://doi. org/10.1016/j.humimm.2021.01.007.

14. Ferrara, F., \& Vitiello, A. (2021). Efficacy of synthetic glucocorticoids in COVID-19 endothelites. Naunyn-Schmiedeberg's Archives of Pharmacology. https://doi.org/10.1007/ s00210-021-02049-7.

15. Vitiello, A., Ferrara, F., \& Porta, R. (2021). Remdesivir and COVID-19 infection, therapeutic benefits or unnecessary risks? Irish Journal of Medical Science, 12, 1-2. https://doi.org/10.1007/ s11845-020-02482-2.

16. Yamamoto, S., Yancey, P. G., Zuo, Y., Ma, L. J., Kaseda, R., Fogo, A. B., Ichikawa, I., Linton, M. F., Fazio, S., \& Kon, V. (2011). Macrophage polarization by angiotensin II-type 1 receptor aggravates renal injury-acceleration of atherosclerosis. Arteriosclerosis,
Thrombosis, and Vascular Biology, 31(12), 2856-2864. https:// doi.org/10.1161/ATVBAHA.111.237198.

17. Recinos, A., 3rd., LeJeune, W. S., Sun, H., Lee, C. Y., Tieu, B. C., Lu, M., Hou, T., Boldogh, I., Tilton, R. G., \& Brasier, A. R. (2007). Angiotensin II induces IL-6 expression and the JakSTAT3 pathway in aortic adventitia of LDL receptor-deficient mice. Atherosclerosis, 194(1), 125-133. https://doi.org/10.1016/j. atherosclerosis.2006.10.013.

18. Pai, W. Y., Lo, W. Y., Hsu, T., Peng, C. T., \& Wang, H. J. (2017). Angiotensin-(1-7) inhibits thrombin-induced endothelial phenotypic changes and reactive oxygen species production via NADPH oxidase 5 downregulation. Frontiers in Physiology, 8(8), 994. https://doi.org/10.3389/fphys.2017.00994.

19. Magalhães, G. S., Rodrigues-Machado, M. G., \& Motta-Santos, D. (2015). Angiotensin-(1-7) attenuates airway remodelling and hyperresponsiveness in a model of chronic allergic lung inflammation. British Journal of Pharmacology., 172(9), 2330-2342. https://doi.org/10.1111/bph.13057.

20. Vitiello, A., \& Ferrara, F. (2020). Correlation between renin-angiotensin system and severe acute respiratory syndrome coronavirus 2 infection: What do we know? European Journal of Pharmacology, 883, 173373. https://doi.org/10.1016/j.ejphar.2020.173373.

21. Vitiello, A., \& Ferrara, F. (2021). Therapeutic Strategies for SARS-CoV-2 acting on ACE-2. European Journal of Pharmaceutical Sciences, 156, 105579. https://doi.org/10.1016/j.ejps. 2020.105579.

22. Zhang, H., Penninger, J. M., Li, Y., Zhong, N., \& Slutsky, A. S. (2020). Angiotensin-converting enzyme 2 (ACE2) as a SARSCoV-2 receptor: molecular mechanisms and potential therapeutic target. Intensive Care Medicine, 46(4), 586-590. https://doi.org/ 10.1007/s00134-020-05985-9.

23. Liu, Y., Yang, Y., Zhang, C., Huang, F., Wang, F., Yuan, J., et al. (2020). Clinical and biochemical indexes from 2019$\mathrm{nCoV}$ infected patients linked to viral loads and lung injury. Sci China Life Sci., 63(3), 364-374. https://doi.org/10.1007/ s11427-020-1643-8.

24. Zou, X., Chen, K., Zou, J., Han, P., Hao, J., \& Han, Z. (2020). Single-cell RNA-seq data analysis on the receptor ACE2 expression reveals the potential risk of different human organs vulnerable to 2019-nCoV infection. Frontiers in Medicine, 14(2), 185-192. https://doi.org/10.1007/s11684-020-0754-0.

25. Zhang J, Wu Y, Wang R, Lu K, Tu M,GuoH et al. (2020) Bioinformatic analysis reveals that the reproductive system is potentially at risk from SARS-CoV-2 doi: https://doi.org/10.20944/preprints2 02002.0307.v1

26. Lukassen, S., Chua, R. L., Trefzer, T., Kahn, N. C., Schneider, M. A., Muley, T., et al. (2020). SARS-CoV-2 receptor ACE2 and TMPRSS 2 are primarily expressed in bronchial transient secretory cells. EMBO Journal, 39(10), e105114. https://doi.org/10.15252/ embj.20105114.

27. Su, H., Yang, M., Wan, C., Yi, L. X., Tang, F., Zhu, H. Y., Yi, F., Yang, H. C., Fogo, A. B., Nie, X., \& Zhang, C. (2020). Renal histopathological analysis of 26 postmortem findings of patients with COVID-19 in China. Kidney International, 98(1), 219-227. https://doi.org/10.1016/j.kint.2020.04.003.

28. Pan, X. W., Xu, D., Zhang, H., Zhou, W., Wang, L. H., \& Cui, X. G. (2020). Identification of a potential mechanism of acute kidney injury during the COVID-19 outbreak: a study based on single-cell transcriptome analysis. Intensive Care Medicine, 46(6), 1114-1116. https://doi.org/10.1007/s00134-020-06026-1.

29. Puelles, V. G., Lütgehetmann, M., Lindenmeyer, M. T., Sperhake, J. P., Wong, M. N., Allweiss, L., et al. (2020). Multiorgan and renal tropism of SARS-CoV-2. New England Journal of Medicine., 383, 590-592.

30. Douglas, G. C., O’Bryan, M. K., Hedger, M. P., Lee, D. K. L., Yarski, M. A., Smith, A. I., \& Lew, R. A. (2004). The novel 
angiotensin-converting enzyme (ACE) homolog, ACE2, is selectively expressed by adult Leydig cells of the testis. Endocrinology, $145,4703-4711$.

31. Li, Y., Xu, Q., Ma, L., Wu, D., Gao, J., Chen, G., \& Li, H. (2020). Systematic profiling of ACE2 expression in diverse physiological and pathological conditions for COVID-19/SARS-CoV-2. Journal of Cellular and Molecular Medicine., 24, 9478-9948.

32. Pinto, B. G. G., Oliveira, A. E. R., Singh, Y., Jimenez, L., Goncalves, A. N. A., Ogava, R. L. T., et al. (2020). ACE2 expression is increased in the lungs of patients with comorbidities associated with severe COVID-19. The Journal of Infectious Diseases, 222, 556-563.

33. Higham, A., \& Singh, D. (2020). Increased ACE2 Expression in Bronchial Epithelium of COPD Patients who are Overweight. Obesity (Silver Spring), 28(9), 1586-1589. https://doi.org/10. 1002/oby.22907.

34. Vitiello, A., La Porta, R., \& Ferrara, F. (2021). Scientific hypothesis and rational pharmacological for the use of sacubitril/valsartan in cardiac damage caused by COVID-19. Medical Hypotheses, 147,110486

35. Vitiello, A., La Porta, R., D’Aiuto, V., \& Ferrara, F. (2021). The risks of liver injury in COVID-19 patients and pharmacological management to reduce or prevent the damage induced. Egypt Liver Journal., 11, 11. https://doi.org/10.1186/s43066-021-00082-y.

36. Ferrara, F., \& Vitiello, A. (2020). Potential pharmacological approach in the regulation of ACE-2 and DPP-IV in diabetic COVID-19 patient. Italian Journal of Medicine. https://doi.org/ 10.4081/itjm.2020.1435.

37. Vitiello, A., Pelliccia, C., \& Ferrara, F. (2021). Drugs acting on the renin-angiotensin system and SARS-CoV-2. Drug Discovery Today, S1359-6446(21), 00037-00044. https://doi.org/10.1016/j. drudis.2021.01.010.

38. Li, X., Rayford, H., \& Uhal, B. D. (2003). Essential roles for angiotensin receptor AT1a in bleomycin-induced apoptosis and lung fibrosis in mice. American Journal of Pathology, 163(6), 2523-2530. https://doi.org/10.1016/S0002-9440(10)63607-3.

39. Deng, J., Wang, D. X., Deng, W., Li, C. Y., \& Tong, J. (2012). The effect of endogenous angiotensin II on alveolar fluid clearance in rats with acute lung injury. Canadian Respiratory Journal, 19(5), 311-318. https://doi.org/10.1155/2012/951025.

40. Shen, L., Mo, H., Cai, L., Kong, T., Zheng, W., Ye, J., Qi, J., \& Xiao, Z. (2009). Losartan prevents sepsis-induced acute lung injury and decreases activation of nuclear factor kappaB and mitogen-activated protein kinases. Shock, 31(5), 500-506. https://doi. org/10.1097/SHK.0b013e318189017a.

41. Caldeira, D., Alarcão, J., Vaz-Carneiro, A., \& Costa, J. (2012). Risk of pneumonia associated with use of angiotensin converting enzyme inhibitors and angiotensin receptor blockers: systematic review and meta-analysis. $B M J, 11(345)$, e4260. https://doi.org/ 10.1136/bmj.e4260.

42. Shrikrishna, D., Astin, R., Kemp, P. R., \& Hopkinson, N. S. (2012). Renin-angiotensin system blockade: a novel therapeutic approach in chronic obstructive pulmonary disease. Clinical Science (London, England), 123(8), 487-498. https://doi.org/10. 1042/CS20120081.

43. Wang, R., Ramos, C., Joshi, I., Zagariya, A., Pardo, A., Selman, M., \& Uhal, B. D. (1999). Human lung myofibroblast-derived inducers of alveolar epithelial apoptosis identified as angiotensin peptides. American Journal of Physiology, 277(6), L1158-L1164. https://doi.org/10.1152/ajplung.1999.277.6.L1158.

44. Wang, R., Zagariya, A., Ibarra-Sunga, O., Gidea, C., Ang, E., Deshmukh, S., Chaudhary, G., Baraboutis, J., Filippatos, G., \& Uhal, B. D. (1999). Angiotensin II induces apoptosis in human and rat alveolar epithelial cells. American Journal of Physiology,
276(5), L885-L889. https://doi.org/10.1152/ajplung.1999.276.5. L885.

45. Papp, M., Li, X., Zhuang, J., Wang, R., \& Uhal, B. D. (2002). Angiotensin receptor subtype AT(1) mediates alveolar epithelial cell apoptosis in response to ANG II. American Journal of Physiology. Lung Cellular and Molecular Physiology, 282(4), L713L718. https://doi.org/10.1152/ajplung.00103.2001.

46. Wang, L., Wang, Y., Yang, T., Guo, Y., \& Sun, T. (2015). Angiotensin-converting enzyme 2 attenuates bleomycin-induced lung fibrosis in mice. Cellular Physiology and Biochemistry, 36(2), 697-711. https://doi.org/10.1159/000430131.

47. Meng, Y., Yu, C. H., Li, W., Li, T., Luo, W., Huang, S., Wu, P. S., Cai, S. X., \& Li, X. (2014). Angiotensin-converting enzyme 2/ angiotensin-(1-7)/Mas axis protects against lung fibrosis by inhibiting the MAPK/NF- $\mathrm{\kappa B}$ pathway. American Journal of Respiratory Cell and Molecular Biology, 50(4), 723-736. https://doi.org/ 10.1165/rcmb.2012-0451OC.

48. Xu, J., Huang, C., Fan, G., et al. (2020). Use of angiotensinconverting enzyme inhibitors and angiotensin II receptor blockers in context of COVID-19 outbreak: a retrospective analysis. Frontiers in Medicine, 14(5), 601-612. https://doi.org/10.1007/ s11684-020-0800-y.

49. Khan, A., Benthin, C., Zeno, B., Albertson, T. E., Boyd, J., Christie, J. D., Hall, R., Poirier, G., Ronco, J. J., Tidswell, M., Hardes, K., Powley, W. M., Wright, T. J., Siederer, S. K., Fairman, D. A., Lipson, D. A., Bayliffe, A. I., \& Lazaar, A. L. (2017). A pilot clinical trial of recombinant human angiotensin-converting enzyme 2 in acute respiratory distress syndrome. Critical Care, 21(1), 234. https://doi.org/10.1186/s13054-017-1823-x.

50. Vitiello, A., \& Ferrara, F. (2020). Pharmacological agents to therapeutic treatment of cardiac injury caused by Covid-19. Life Sciences, 1(262), 118510. https://doi.org/10.1016/j.lfs.2020.118510.

51. Vitiello, A., La Porta, R., \& Ferrara, F. (2020). Sacubitril valsartan and SARS-CoV-2. BMJ Evid Based Med. https://doi.org/10.1136/ bmjebm-2020-111497.

52. Zhang, H., Penninger, J. M., Li, Y., Zhong, N., \& Slutsky, A. S. (2020). Angiotensin-converting enzyme 2 (ACE2) as a SARS$\mathrm{CoV}-2$ receptor molecular mechanisms and potential therapeutic target. Intensive Care Medicine, 46, 586-590.

53. Monteil, V., Kwon, H., Prado, P., et al. (2020). Inhibition of SARS-CoV-2 infections in engineered human tissues using clinical-grade soluble human ACE2. Cell, 181, 905-913.

54. Kuba, K., Imai, Y., Rao, S., et al. (2005). A crucial role of angiotensin converting enzyme 2 (ACE2) in SARS coronavirus-induced lung injury. Nature Medicine, 11, 875-879.

55. Haschke, M., Schuster, M., Poglitsch, M., et al. (2013). Pharmacokinetics and pharmacodynamics of recombinant human angiotensin-converting enzyme 2 in healthy human subjects. Clinical Pharmacokinetics, 52, 783-792.

56. Khan, A., Benthin, C., \& Zeno, B. (2017). A pilot clinical trial of recombinant human angiotensin-converting enzyme 2 in acute respiratory distress syndrome. Critical Care, 21, 234.

57. Minato, T., Nirasawa, S., Sato, T., Yamaguchi, T., Hoshizaki, M., Inagaki, T., et al. (2020). B38-CAP is a bacteria-derived ACE2like enzyme that suppresses hypertension and cardiac dysfunction. Nature Communications, 11(1), 1058. https://doi.org/10.1038/ s41467-020-14867-z.

Publisher's Note Springer Nature remains neutral with regard to jurisdictional claims in published maps and institutional affiliations. 